\title{
BIRATIONAL RIGIDITY AND K-STABILITY OF FANO HYPERSURFACES WITH ORDINARY DOUBLE POINTS
}

\author{
TOMMASO DE FERNEX
}

\begin{abstract}
Extending previous results, we prove that for $n \geq 5$ all hypersurfaces of degree $n+1$ in $\mathbb{P}^{n+1}$ with isolated ordinary double points are birational superrigid and K-stable, hence admit a weak Kähler-Einstein metric.
\end{abstract}

\section{INTRODUCTION}

This work continues prior research on birational superrigidity of Fano hypersurfaces in projective spaces, a property of certain Mori fiber spaces that was first identified for smooth quartic threefolds as a byproduct of Iskovskikh and Manin's proof of nonrationality.

The starting point of the paper is the following theorem.

Theorem 1.1. For $n \geq 3$, every smooth hypersurface of degree $n+1$ in $\mathbb{P}^{n+1}$ is birationally superrigid, hence nonrational.

This theorem has a long history that covers over a century, starting with the work of Fano on quartic threefolds [Fan07, Fan15] which was later revisited and completed by Iskovskikh and Manin [IM71]. The higher dimensional case was promoted in the work of Pukhlikov [Puk87, Puk98], where significant advances were made, and a complete proof was eventually given in [dF16]; the proof was later simplified in [Zhu20]. Other people have contributed with key ideas along the way; we recall here Segre and Manin's works of cubic surfaces over nonclosed fields [Seg51, Man66], Corti's new proof of the three-dimensional case where inversion of adjunction is used for the first time to connect the method of maximal singularities to log canonical thresholds [Cor00], and the author's work with Ein and Mustaţă on log canonical thresholds [dFEM03,dFEM04]. A survey of the history of Theorem 1.1 can be found in [Kol19]. We will briefly review the proof of Theorem 1.1 following [Zhu20, Kol19] before turning our attention to the case of singular hypersurfaces.

Birational superrigidity is sensitive to singularities. Singular quartic threefolds in $\mathbb{P}^{4}$ are never birationally superrigid, though under some reasonable assumptions those with ordinary double points are birational rigid, which is a weaker condition compared to superrigidity [Puk88, CM04, Mel04]. In higher dimensions, Pukhlikov extended his proof of birational superrigidity of general smooth Fano hypersurfaces to general hypersurfaces with isolated ordinary multiple points [Puk02b], and with Eckl, they prove a similar result for general hypersurfaces with quadratic (non-necessarily isolated) singularities of rank $\geq 5$ [EP14]. Cheltsov looked at sextic fivefolds in $\mathbb{P}^{6}$ with isolated ordinary double points, proving that they are always birationally superrigid provided they do not contain any plane [Che07].

2020 Mathematics Subject Classification. Primary: 14J45, 14E08; Secondary: 32Q20.

Key words and phrases. Birational rigidity, K-stability.

The research of the first author was partially supported by NSF Grant DMS-2001254. 
More recently, birational superrigidity of singular hypersurfaces was established in [dF17] under rather broad assumptions on the singularities, where a certain measure of the singularity is bounded in terms of the dimension. An asymptotically stronger bound was later given under more restrictive conditions on the types of singularities in [LZ21]. While these results are asymptotically strong as the dimension grows, they leave a gap open in low dimensions.

Even restricting to the simplest case of isolated ordinary double points (namely, points whose tangent cones are cones over smooth quadrics), the main theorem of [dF17] only covers the cases of dimension $n \geq 19$, leaving Cheltsov's theorem on sextic fivefolds not containing planes and Pukhlikov's result for general hypersurfaces with isolated ordinary double points the only known results in the remaining cases $4 \leq n \leq 18$.

The purpose of this paper is to try to fill this gap.

Theorem 1.2. For $n \geq 5$, every hypersurface of degree $n+1$ in $\mathbb{P}^{n+1}$ with only isolated ordinary double points as singularities is birationally superrigid, hence nonrational.

To prove this result, we provide a new asymptotic argument which allows us to cover all cases $n \geq 8$, thus reducing the gap to a few lower dimensional cases. The remaining cases are treated using an extension to singular hypersurfaces of the projection method of [dFEM03], which is made possible using a result from [dFM15] on Mather log discrepancies. The proof does not extend to the case $n=4$, and the question whether all quintic hypersurfaces in $\mathbb{P}^{5}$ with isolated ordinary double points are birationally superrigid remains open.

Birational superrigidity is a property closely related to K-stability. A first indication of this was discovered in [OO13], and a more precise connection was established in [SZ19, Zhu20]. It was observed in particular in [SZ19] that Theorem 1.1, combined with Cheltsov's bound on the $\alpha$-invariant [Che01], implies that all smooth hypersurfaces of degree $n+1$ in $\mathbb{P}^{n+1}$ are K-stable, hence admit a Kähler-Einstein metric. Note that the same conclusion follows from by combining Cheltsov's bound to [Fuj19b], which extends Tian's criterion for K-stability of Fano manifolds [Tia87] to the boundary case.

Under suitable generality conditions on the defining equation which are met by general hypersurfaces of degree $n+1$ in $\mathbb{P}^{n+1}$ with quadratic singularities of rank $\geq 8$, Pukhlikov established stronger bounds on $\alpha$-invariant and canonical thresholds, see [Puk05, Puk15, Puk21]. These bounds immediately imply K-stability by Tian's criterion and its extension to singular Fano varieties due to [OS12]; note, however, that these stronger bounds do not holds without some generality conditions. See also [LZ19] regarding the sharpness of Tian's criterion and its aforementioned extensions.

Here we apply [SZ19], along with [LXZ21], to deduce the following result from Theorem 1.2.

Theorem 1.3. For $n \geq 5$, every hypersurface of degree $n+1$ in $\mathbb{P}^{n+1}$ with only isolated ordinary double points as singularities is K-stable and hence admits a weak Kähler-Einstein metric.

Acknowledgements. We would like to thank Aleksandr Pukhlikov for bringing to our attention several relevant references, Kento Fujita and Yuga Tsubouchi for pointing out an error in the proof of an earlier version of Lemma 2.17, and the referee for valuable comments and for suggesting an extension of Theorem 1.2 to quadratic singularities of rank $\geq 2$ (see Remark 3.2). 


\section{Preliminaries on Singularities}

We work over an algebraically closed field of characteristic zero.

2.1. Multiplicities. We start this section by recalling some basic properties of multiplicities. We follow [Ful98]. In particular, for us the multiplicity $e_{p}(X)$ of a pure dimensional scheme $X$ at a closed point $p$ is defined to be the Samuel multiplicity $e\left(\mathfrak{m}_{p}\right)$ of the maximal ideal $\mathfrak{m}_{p}$ of the local ring $\mathcal{O}_{X, p}$.

Example 2.1. If $X \subset \mathbb{A}^{n}$ is a pure dimensional Cohen-Macaulay scheme and $p \in X$ is a closed point, then $e_{p}(X)=i\left(p, X \cdot L ; \mathbb{A}^{n}\right)$, the intersection multiplicity of $X$ with a general linear subspace $L \subset \mathbb{A}^{n}$ of codimension equal to the dimension of $X$. This follows from [Ful98, Example 4.3.5(c) and Proposition 7.1]. It relates the definition of multiplicity adopted here with more classical definitions. The equality does not hold in general if $X$ is not Cohen-Macaulay.

Example 2.2. If $D$ is an effective Cartier divisor on a variety $X$ and $p \in X$ is a regular point, then $e_{p}(D)$ is simply the order of vanishing of $D$ at $p$, namely, the multiplicity of a generator of the ideal of $D$ in the local ring $\mathcal{O}_{X, p}$ [Ful98, Example 4.3.9]. This is no longer true, however, if $p$ is a singular point of $X$ (cf. Remark 2.5 below).

More generally, for any closed subscheme $Z$ of a pure dimensional scheme $X$ and any irreducible component $T$ of $Z$, the multiplicity of $X$ along $Z$ at $T$, denoted by $e_{Z}(X)_{T}$, is defined to be the Samuel multiplicity of the ideal of $Z$ in the local ring $\mathcal{O}_{X, T}$; equivalently, $e_{Z}(X)_{T}$ is the coefficient of $[T]$ in the Segre class of the normal cone to $Z$ in $X$ [Ful98, Example 4.3.4]. If $Z=T$, then we just write $e_{T}(X)$.

Example 2.3. Whenever $T$ is an irreducible component of a scheme $Z$ we have $e_{T}(Z)=$ $l\left(\mathcal{O}_{Z, T}\right)$ by definition, and if $Z$ is regularly embedded in a pure dimensional CohenMacaulay scheme $X$ then $e_{T}(Z)=e_{Z}(X)_{T}$ [Ful98, Example 4.3.5(c)].

Example 2.4. If $Z=D_{1} \cap \cdots \cap D_{r} \subset X$ is the complete intersection of $r$ divisors on variety $X$ and $T$ is an irreducible component of $Z$, then $e_{Z}(X)_{T}=i\left(T, D_{1} \cdot \ldots \cdot D_{r} ; X\right)$, where the last term denotes the intersection multiplicity of the divisors $D_{i}$ at the generic point of $T$ [Ful98, Example 7.1.10.(a)].

Multiplicities are semicontinuous, in the following sense. If $X$ is a pure-dimensional scheme, then for every irreducible closed set $T \subset X$ there is a nonempty open set $T^{\circ} \subset T$ such that $e_{p}(X) \geq e_{T}(X)$ for every point $p \in X$, and equality holds if $p \in T^{\circ}$. This is proved in [Ben70, Theorem (4)]; we will tacitly use this fact without further mention.

Remark 2.5. Differently from the multiplicity, the order of vanishing of a Cartier divisor $D$ on a variety $X$ is not always a semicontinuous function of the point of the variety. For example, take $X \subset \mathbb{A}^{3}$ to be a cone over a smooth conic and $D=2 L$ where $L$ is a line through the vertex of the cone. The order of vanishing $D$ at any point of $L$ is 2 except at the vertex, where the order of vanishing drops to 1 .

We list a few properties of multiplicities that will be used later in the paper. The first property, which can be interpreted as a Bertini-type property, is proved in [dFEM03, Proposition 4.5] and [dF13, Proposition 8.5].

Proposition 2.6. Let $Z$ be a pure-dimensional closed Cohen-Macaulay subscheme of $\mathbb{P}^{m}$ of positive dimension and $\mathcal{H} \subset\left(\mathbb{P}^{m}\right)^{\vee}$ a hyperplane in the dual space. Then for a general $H \in \mathcal{H}$ we have $e_{p}(Z \cap H)=e_{p}(Z)$ for every $p \in Z \cap H$. 
The proof of the next Bezout inequality combines various properties established in [Ful98] and is included for the convenience of the reader.

Proposition 2.7. Let $Z=X \cap H_{1} \cap \cdots \cap H_{r}$ be the complete intersection of a variety $X \subset \mathbb{P}^{n}$ with $r$ hypersurfaces $H_{i} \subset \mathbb{P}^{n}$. Then for every irreducible component $T$ of $Z$ we have

$$
e_{Z}(X)_{T} \leq \operatorname{deg} X \cdot \prod \operatorname{deg} H_{i}
$$

Proof. As we recalled in Example 2.4, letting $D_{i}=H_{i} \cap X$ we have $e_{Z}(X)_{T}=i\left(T, D_{1}\right.$. $\left.\ldots \cdot D_{r} ; X\right)$. By the definition of intersection product, this number is bounded above by the intersection product $\left(D_{1} \cdot \ldots \cdot D_{r}\right)_{X}$, which in turns is equal to $\left(H_{1} \cdot \ldots \cdot H_{r}\right.$. $[X])_{\mathbb{P}^{n}}$, see [Ful98, Example 2.4.3]. Therefore the proposition follows by Bezout theorem [Ful98, Propostion 8.4].

Finally, we recall the following multiplicity bound on cycles on projective hypersurfaces due to [Puk02a, Proposition 5] (cf. [dFEM03, Remark 4.4]).

Proposition 2.8. Let $X \subset \mathbb{P}^{n}$ be a smooth hypersurface and $Z \subset X$ a pure-dimensional closed scheme with no embedded points. Let $k=\operatorname{codim}(Z, X)$, and assume that the cycle $[Z]$ is numerically equivalent to $m \cdot c_{1}\left(\mathcal{O}_{X}(1)\right)^{k}$. Then $\operatorname{dim}\left\{x \in X \mid e_{x}(Z)>m\right\}<k$.

Remark 2.9. The example discussed in Remark 2.5 shows that Proposition 2.8 does not hold in general if the hypersurface is singular. However, one can always cut down away from the singularities and use Proposition 2.6 to generalize the proposition to singular hypersurfaces. In particular, if $X \subset \mathbb{P}^{n}$ is a hypersurface with isolated singularities then $\operatorname{dim}\left\{x \in X \mid e_{x}(Z)>m\right\} \leq k$, and we have $e_{T}(Z) \leq m$ for every closed subvariety $T \subset X$ of dimension $k$ that is disjointed from the singular locus of $X$.

2.2. Singularities of pairs. Let $X$ be a positive dimensional normal variety and assume that the canonical divisor $K_{X}$ of $X$ is $\mathbb{Q}$-Cartier.

We consider pairs of the form $(X, Z)$ where $Z=\sum c_{i} Z_{i}$ is a $\mathbb{Q}$-subscheme, i.e., a proper linear combination of proper closed subschemes $Z_{i} \subset X$ with coeffients $c_{i}$ in $\mathbb{Q}$. We say that the $\mathbb{Q}$-subscheme $Z$ (or the pair $(X, Z)$ ) is effective if $c_{i} \geq 0$ for all $i$. If $\mathcal{H}$ is a linear system on $X$, then we denote by $(X, c \mathcal{H})$ the pair $(X, c \operatorname{Bs}(\mathcal{H}))$ where $\operatorname{Bs}(\mathcal{H})$ is the base scheme of $\mathcal{H}$. We will also consider pairs of the form $\left(X, \mathfrak{a}^{c}\right)$ where $\mathfrak{a} \subset \mathcal{O}_{X}$ is a nonzero coherent ideal sheaf and $c \in \mathbb{Q}$, and $(X, \Delta)$ where $\Delta$ is a $\mathbb{Q}$-Cartier $\mathbb{Q}$-divisor; both versions can be view in an obvious way as special cases of the above definition of pair.

We will refer to any prime divisor $E$ on a resolution of $X$ as a divisor over $X$, and denote by $c_{X}(E) \subset X$ the closure of the center of the corresponding valuation val $E$. With small abuse of terminology, we call $c_{X}(E)$ the center of $E$ in $X$, and identify two divisors over $X$ whenever they define the same valuation.

Given a pair $(X, Z)$ and a divisor $E$ over $X$, we define the log discrepancy of $(X, Z)$ along $E$ to be

$$
a_{E}(X, Z):=\operatorname{ord}_{E}\left(K_{Y / X}\right)+1-\operatorname{val}_{E}(Z),
$$

where $Y$ is a resolution of $X$ where $E$ lives, $K_{Y / X}$ is the relative canonical divisor (a $\mathbb{Q}$-divisor), and $\operatorname{val}_{E}(Z):=\sum c_{i} \operatorname{val}_{E}\left(\mathcal{I}_{Z_{i}}\right)$ where $\mathcal{I}_{Z_{i}} \subset \mathcal{O}_{X}$ is the ideal sheaf of $Z_{i}$. If $Z=0$, then we simply write $a_{E}(X)$ (the same quantity is also denoted by $A_{X}(E)$ in the literature). The minimal log discrepancy $\operatorname{mld}_{p}(X, Z)$ of a pair $(X, Z)$ at a point $p \in X$ is the infimum of the $\log$ discrepancies $a_{E}(X, Z)$ of all divisors $E$ over $X$ with center $p$. 
Remark 2.10. Once $E$ is fixed, if $\mathcal{H}$ is a linear system and $D_{i} \in \mathcal{H}$ are general members of it, then $a_{E}(X, \mathcal{H})=a_{E}\left(X, \cap D_{i}\right)$. Using resolution of singularities, one also sees that once a point $p \in X$ is fixed, if $D_{i} \in \mathcal{H}$ are general members then $\operatorname{mld}_{p}(X, \mathcal{H})=\operatorname{mld}_{p}\left(X, \bigcap D_{i}\right)$.

The next property is an immediate consequence of Shokurov-Kollár's connectedness principle [Sho92, Kol92].

Theorem 2.11 (Inversion of Adjunction). Let $(X, Z)$ be an effective pair where $Z=$ $\sum c_{i} Z_{i}$, let $H \subset X$ be a normal irreducible Cartier divisor that is not contained in any $Z_{i}$, and set $\left.Z\right|_{H}:=\sum c_{i}\left(Z_{i} \cap H\right)$. Suppose $p \in H$ is a closed point where $\operatorname{mld}_{p}(X, Z+H) \leq 0$. Then $\operatorname{mld}_{p}\left(H,\left.Z\right|_{H}\right) \leq 0$.

Remark 2.12. The converse of Theorem 2.11 also holds, namely, we have $\operatorname{mld}_{p}(X, Z+H) \leq$ 0 whenever $\operatorname{mld}_{p}\left(H,\left.Z\right|_{H}\right) \leq 0$. This direction is a simple consequence of the adjunction formula.

Assuming $\operatorname{dim} X \geq 2$, a pair $(X, Z)$ is said to be terminal (resp., canonical) if $a_{E}(X, Z)>$ 1 (resp., $\geq 1$ ) for all exceptional divisors $E$ over $X$. A pair $(X, Z)$ is said to be log terminal (resp., $\log$ canonical) if $a_{E}(X, Z)>0$ (resp., $\geq 0$ ) for all divisors $E$ over $X$.

Let $(X, Z)$ be an effective pair. If $\operatorname{dim} X \geq 2$ and $X$ is canonical, then we denote by $\operatorname{ct}(X, Z)$ the canonical threshold, namely, the supremum of the set of coefficients $c$ such that $(X, c Z)$ is canonical. If $X$ is $\log$ canonical, then we $\operatorname{denote}$ by $\operatorname{lct}(X, Z)$ the $\log$ canonical threshold, namely, the supremum such that $(X, c Z)$ is $\log$ canonical. For a point $p \in X$, we denote by $\operatorname{ct}_{p}(X, Z)$ and $\operatorname{lct}_{p}(X, Z)$ the canonical and log canonical thresholds of the pair restricted to any sufficiently small open neighborhood of $p$.

The next proposition relates thresholds to multiplicities. The first property stated in the proposition follows from inversion of adjunction (e.g., see [dF13, Proposition 8.8]). The second is proved in [dFEM04].

Proposition 2.13. Let $X$ be a smooth $n$-dimensional variety and $p \in X$ a closed point.

(1) If $n \geq 2$, then for any effective divisor $D$ on $X$, we have $e_{p}(D) \geq 1 / \operatorname{ct}_{p}(X, D)$.

(2) For any zero-dimensional subscheme $Z$ of $X$, we have $e_{Z}(X)_{p} \geq n^{n} / \operatorname{lct}_{p}(X, Z)^{n}$.

The multiplier ideal of an effective pair $(X, Z)$, where $Z=\sum c_{i} Z_{i}$, is defined by taking a $\log$ resolution $f: Y \rightarrow X$ of the pair and setting

$$
\mathcal{J}(X, Z):=f_{*}\left(\left\lceil K_{Y / X}-f^{-1}(Z)\right\rceil\right)
$$

where $f^{-1}(Z)$ denotes the $\mathbb{Q}$-divisor $\sum c_{i} f^{-1}\left(Z_{i}\right)$. It is a standard computation to check that the definition is independent of the choice of resolution. Multiplier ideals satisfy the following vanishing theorem due to Nadel (see [Laz04, Section 9.4]).

Theorem 2.14 (Nadel Vanishing Theorem). Let $(X, Z)$ be an effective pair with $Z=$ $\sum c_{i} Z_{i}$, let $A_{i}$ be Cartier divisors on $X$ such that $\mathcal{O}_{X}\left(A_{i}\right) \otimes \mathcal{I}_{Z_{i}}$ is globally generated for every $i$, and let $A$ be a Cartier divisor such that the $\mathbb{Q}$-divisor $A-K_{X}-\sum c_{i} A_{i}$ is nef and big. Then $H^{j}\left(X, \mathcal{O}_{X}(A) \otimes \mathcal{J}(X, Z)\right)=0$ for all $j \geq 1$.

2.3. Bounds on colength. Here we review the main new ingredient of [Zhu20] as revisited in [Kol19].

Proposition 2.15. Let $X$ be a smooth variety of dimension $n \geq 2$, and $Z$ an effective $\mathbb{Q}$-scheme on $X$ with $\operatorname{lct}(X, Z) \leq 1$. If $\Sigma \subset X$ is the subscheme defined by the multiplier ideal $\mathcal{J}(X, 2 Z) \subset \mathcal{O}_{X}$, then

$$
l\left(\mathcal{O}_{\Sigma}\right) \geq \frac{1}{2} 3^{n}
$$


We briefly review the proof. We start with two lemmas.

Lemma 2.16. Let $(X, Z)$ be an effective pair. Then for every divisor $E$ over $X$ we have

$$
a_{E}(X, \mathcal{J}(X, 2 Z))<2 a_{E}(X, Z) .
$$

In particular, if $(X, Z)$ is not log terminal, then $(X, \mathcal{J}(X, 2 Z))$ is not log canonical.

Proof. Denote for short $\mathcal{J}=\mathcal{J}(X, 2 Z)$. We may assume that $E$ is a prime divisor on a $\log$ resolution $f: Y \rightarrow X$ of $(X, Z)$. Set $k_{E}=\operatorname{ord}_{E}\left(K_{Y / X}\right), z_{E}=\operatorname{ord}_{E}(Z)$, and $j_{E}=\operatorname{ord}_{E}(\mathcal{J})$. By definition, $\mathcal{J}=f_{*} \mathcal{O}_{Y}\left(-\left\lfloor 2 f^{-1}(Z)-K_{Y / X}\right\rfloor\right)$, hence

$$
j_{E} \geq \operatorname{ord}_{E}\left(\left\lfloor 2 f^{-1}(Z)-K_{Y / X}\right\rfloor\right)=\left\lfloor 2 z_{E}-k_{E}\right\rfloor>2 z_{E}-k_{E}-1 .
$$

This implies that

$$
a_{E}(X, \mathcal{J})=k_{E}+1-j_{E}<2 k_{E}+2-2 z_{E}=2 a_{E}(X, Z),
$$

as claimed.

Lemma 2.17. Let $R=\mathcal{O}_{X, x}$ be the local ring of a variety $X$ at a regular point $x$. Assume that $\operatorname{dim} R=n \geq 2$, and let $\mathfrak{a} \subset R$ be an $\mathfrak{m}$-primary ideal with $\operatorname{lct}(\mathfrak{a})<1$. Then

$$
l(R / \mathfrak{a}) \geq \frac{1}{2} 3^{n}
$$

Proof. A standard argument (see, e.g., the proof of [dFEM04, Theorem 1.1]) allows to reduce to the case where $\mathfrak{a}$ is a monomial ideal in a polynomial ring $k\left[x_{1}, \ldots, x_{n}\right]$. The condition that $\operatorname{lct}(\mathfrak{a})<1$ implies that there are positive numbers $a_{i}$ such that the tetrahedron

$$
T=\left\{u \in \mathbb{R}_{\geq 0}^{n} \mid \sum a_{i} u_{i} \leq \sum a_{i}\right\}
$$

is disjoint from the Newton polyhedron $P(\mathfrak{a})$. Hence $l(R / \mathfrak{a}) \geq\left|T \cap \mathbb{Z}^{n}\right|$. The upperbound $\frac{1}{2} 3^{n}$ accounts for the fact that for any $\left(u_{1}, \ldots, u_{n}\right) \in \mathbb{Z}^{n}$ with $u_{i} \in\{0,1,2\}$ for all $i$, either $\left(u_{1}, \ldots, u_{n}\right)$ or $\left(2-u_{1}, \ldots, 2-u_{n}\right)$ belongs to $T$.

Proof of Proposition 2.15. We may assume that $\Sigma$ is zero dimensional as otherwise $l\left(\mathcal{O}_{\Sigma}\right)=$ $\infty$. Since $(X, Z)$ is not log terminal, Lemma 2.16 implies that $(X, \Sigma)$ is not log canonical, that is, $\operatorname{lct}(X, \Sigma)<1$. Then there is a connected component $\Sigma^{\prime}$ of $\Sigma$ such that $\operatorname{lct}\left(X, \Sigma^{\prime}\right)<$ 1 , hence $l\left(\mathcal{O}_{\Sigma^{\prime}}\right)$ satisfies the claimed bound by Lemma 2.17. Since $l\left(\mathcal{O}_{\Sigma}\right) \geq l\left(\mathcal{O}_{\Sigma^{\prime}}\right)$, this proves the proposition.

2.4. Mather log dicrepancies. A different way of measuring singularities of pairs on singular varieties is to use the Jacobian ideal sheaf $\operatorname{Jac}_{f}=$ Fitt $^{0}\left(\Omega_{Y / X}\right)$ of a log resolution $f: Y \rightarrow X$ in place of the relative canonical divisor. We define the Mather log discrepancy of $E$ over a pair $(X, Z)$ to be

$$
\widehat{a}_{E}(X, Z):=\operatorname{ord}_{E}\left(\operatorname{Jac}_{f}\right)+1-\operatorname{ord}_{E}(Z) .
$$

If $X$ is smooth then $\widehat{a}_{E}(X, Z)=a_{E}(X, Z)$, but we have a strict inequality $\widehat{a}_{E}(X, Z)>$ $a_{E}(X, Z)$ as soon as $X$ is singular at the generic point of the center of $E$. This follows from [dFD14, Proposition 3.4], which shows that $\operatorname{ord}_{E}\left(\operatorname{Jac}_{X}\right)=\operatorname{ord}_{E}\left(K_{Y / X}\right)+\frac{1}{r} \operatorname{ord}_{E}\left(\mathfrak{n}_{r, X}\right)$ where $\operatorname{Jac}_{X}=\operatorname{Fitt}^{\operatorname{dim} X}\left(\Omega_{X}\right)$ is the Jacobian ideal sheaf of $X, r$ is any positive integer such that $r K_{X}$ is Cartier, and $\mathfrak{n}_{r, X}$ is the ideal sheaf defined by the image of the natural map $\left(\wedge^{\operatorname{dim} X} \Omega_{X}\right)^{\otimes r} \rightarrow \mathcal{O}_{X}\left(r K_{X}\right)$, and Nobile's theorem [Nob75, Theorem 2], which implies that $\mathfrak{n}_{r, X}$ vanishes precisely on the singular locus of $X$. 
Example 2.18. If $X$ has locally complete intersection singularities, then $\operatorname{ord}_{E}\left(\operatorname{Jac}_{f}\right)=$ $\operatorname{ord}_{E}\left(K_{Y / X}\right)+\operatorname{ord}_{E}\left(\operatorname{Jac}_{X}\right)($ see [Pie79, Proposition 1]), hence

$$
\widehat{a}_{E}(X, Z)=a_{E}(X, Z)+\operatorname{ord}_{E}\left(\operatorname{Jac}_{X}\right) .
$$

Let now $X \subset \mathbb{A}^{N}$ be a Cohen-Macaulay variety of dimension $n$, and $E$ a divisor over $X$. Consider general linear projections

$$
X \subset \mathbb{A}^{N} \stackrel{\psi}{\rightarrow} U=\mathbb{A}^{n} \stackrel{\gamma}{\rightarrow} V=\mathbb{A}^{m}
$$

where $1 \leq m \leq n$. We denote by $\phi: X \rightarrow V$ be the composition. Write $\left.\operatorname{val}_{E}\right|_{k(U)}=p \operatorname{val}_{F}$ and $\left.\operatorname{val}_{E}\right|_{k(V)}=q \operatorname{val}_{G}$ where $F$ and $G$ are divisors over $U$ and $V$, respectively, and $p$ and $q$ are positive integers (e.g., see [dFM15, Lemma 2.3]). Let $Z \subset X$ be a closed subscheme of codimension $r=n-m+1$ cut out by a regular sequence. We assume that $\left.\phi\right|_{Z}$ is a proper finite morphism. Note that $\phi_{*}[Z]$ is a cycle of codimension 1 in $V$. We regard $\phi_{*}[Z]$ as a Cartier divisor on $V$.

Theorem 2.19. With the above notation, for every $c \geq 0$ such that $\widehat{a}_{E}(X, c Z) \geq 0$ we have

$$
q a_{G}\left(V, \frac{c^{r}}{r^{r}} \cdot \phi_{*}[Z]\right) \leq \widehat{a}_{E}(X, c Z) .
$$

This theorem was first proved in [dFEM03] assuming that $X$ is smooth. The proof was then extended to singular varieties using Mather log discrepancies in [dFM15].

\section{Birational Rigidity}

A Mori fiber space is a normal projective variety $X$ with $\mathbb{Q}$-factorial terminal singularities, equipped with an extremal Mori contraction $f: X \rightarrow S$ of fiber type. This means that $f$ is a proper morphism with connected fibers and relative Picard number 1 , the anticanonical class $-K_{X}$ is $f$-ample, and $\operatorname{dim} S<\operatorname{dim} X$. Note that Fano varieties with $\mathbb{Q}$-factorial terminal singularities and Picard number 1 can be viewed as Mori fiber spaces over a point.

A Mori fiber space $f: X \rightarrow S$ is birationally superrigid if for every birational map $\phi: X \rightarrow X^{\prime}$ from $X$ to another Mori fiber space $f^{\prime}: X^{\prime} \rightarrow S^{\prime}$ there exists a birational map $\psi: S \rightarrow S^{\prime}$ such that $f^{\prime} \circ \phi=\psi \circ f$ and, furthermore, $\phi$ induces an isomorphism between the generic fibers of $f$ and $f^{\prime}$.

Birational superrigidity is a notion that is naturally motivated from the point of view of the minimal model program. Clearly, birational superrigid varieties are nonrational. In dimension 2, every rationally connected Mori fiber space is rational and hence cannot be birationally superrigid, something that should be contrasted with the fact that twodimensional minimal models are unique in their birational classes. Both facts are no longer true in higher dimensions. The first example of birationally superrigid rationally connected Mori fiber space was discovered as a byproduct of Iskovskikh and Manin's proof of nonrationality of smooth quartic threefolds in $\mathbb{P}^{4}$.

Restricting to a Fano variety $X$ with $\mathbb{Q}$-factorial terminal singularities and Picard number 1 , we see that $X$ is birationally superrigid if and only if every birational map $\phi: X \rightarrow X^{\prime}$ to a Mori fiber space is an isomorphism. The following result, established in [IM71, Cor00], is at the center of the study of birationally superrigid Fano varieties. A more general version can be stated where $X$ is any Mori fiber space, but we will not need it.

Theorem 3.1 (Noether-Fano Inequality). Let $X$ be a Fano variety with $\mathbb{Q}$-factorial terminal singularities and Picard number 1, and let $\phi: X \rightarrow X^{\prime}$ be a birational map to a 
Mori fiber space $f^{\prime}: X^{\prime} \rightarrow S^{\prime}$. Fix a projective embedding of $X^{\prime}$ given by a linear system $\mathcal{H}^{\prime} \subset\left|-r^{\prime} K_{X^{\prime}}+A^{\prime}\right|$ where $r^{\prime}$ is a positive rational number and $A^{\prime}$ is the pullback of an ample divisor on $S^{\prime}$ (we set $A^{\prime}=0$ if $S^{\prime}$ is a point), so that $\phi$ is defined by the movable linear system $\mathcal{H}:=\phi_{*}^{-1} \mathcal{H}^{\prime}$. Let $r$ the positive rational number such that $\mathcal{H} \subset\left|-r K_{X}\right|$. If $\operatorname{ct}(X, \mathcal{H}) \leq \frac{1}{r}$, then $\phi$ is an isomorphism.

Before addressing Theorem 1.2, we quickly revisit the proof of the smooth case. We follow the proof given in [Zhu20, Kol19].

Proof of Theorem 1.1. Let $X \subset \mathbb{P}^{n+1}$ be a smooth hypersurface of degree $n+1$, with $n \geq 3$. Note that $-K_{X} \sim \mathcal{O}_{X}(1)$, and this generates the Picard group of $X$ by the Lefschetz hyperplane theorem.

We assume by contradiction that $X$ is not birationally superrigid, and let $\phi$ be a birational map to a Mori fiber space $X^{\prime}$ that is not an isomorphism. Let $\mathcal{H} \subset\left|-r K_{X}\right|$ be a linear system defining $\phi$ as in Theorem 3.1. By the Noether-Fano inequality, we have

$$
c:=\operatorname{ct}(X, \mathcal{H})<\frac{1}{r} .
$$

On the other hand, Proposition 2.8 implies that $e_{C}(D) \leq r$ for every $D \in \mathcal{H}$ and every irreducible curve $C \subset X$, hence it follows by Proposition 2.13 that $\left(X, \frac{1}{r} \mathcal{H}\right)$ is canonical in dimension 1 (i.e., away from a finite set). Therefore there is a closed point $p \in X$ such that

$$
\operatorname{mld}_{p}(X, c \mathcal{H})=1 \text {. }
$$

Fix now two general elements $D, D^{\prime} \in \mathcal{H}$, and let $Z=D \cap D^{\prime} \subset X$ their schematic intersection. For any subvariety $V \subset X$, we will denote by $\left.Z\right|_{V}$ the intersection $Z \cap V$. Note that we still have $\operatorname{mld}_{p}(X, c Z)=1$, and Proposition 2.8 implies that the set $\{x \in$ $\left.X \mid e_{x}(Z)>r^{2}\right\}$ is at most one-dimensional. Let $Y \subset X$ be a general hyperplane section through $p$. Bertini's theorem ensures that the pair $\left(Y,\left.c Z\right|_{Y}\right)$ is canonical away from $p$, and inversion of adjunction (Theorem 2.11) implies that

$$
\operatorname{mld}_{p}\left(Y,\left.c Z\right|_{Y}\right) \leq 0 \text {. }
$$

Note that, by Proposition 2.6, the set $\operatorname{dim}\left\{y \in Y \mid e_{y}\left(\left.Z\right|_{Y}\right)>r^{2}\right\}$ is zero-dimensional.

If $n=3$, then $Y$ is a surface and $\left.Z\right|_{Y}$ is zero dimensional. Since $\operatorname{lct}_{p}\left(Y,\left.Z\right|_{Y}\right)<\frac{1}{r}$, we have $e_{\left.Z\right|_{Y}}(Y)_{p}>4 r^{2}$ by Proposition 2.13. On the other hand, observing that $Y$ has degree 4 and $\left.Z\right|_{Y}$ is a zero dimensional complete intersection scheme cut out on $Y$ by two equations of degree $r$, we also have $e_{\left.Z\right|_{Y}}(Y)_{p} \leq 4 r^{2}$ by Proposition 2.7. This gives a contradiction.

Assume therefore that $n \geq 4$.

We clam that the pair $\left(Y,\left.2 c Z\right|_{Y}\right)$ is log terminal in dimension 1 . To see this, first recall that the set $\left\{y \in Y \mid e_{y}\left(\left.Z\right|_{Y}\right)>r^{2}\right\}$ is zero-dimensional, hence there is a finite set $\Gamma \subset Y$ such that $e_{q}\left(\left.Z\right|_{Y}\right) \leq r^{2}$ for all $q \in Y \backslash \Gamma$. For any such $q$, let $S \subset Y$ be a smooth surface cut out by general hyperplanes through $q$. We have $e_{q}\left(\left.Z\right|_{S}\right) \leq r^{2}$, and since $\left.Z\right|_{S}$ is a zero-dimensional complete intersection subscheme of $S$, we have

$$
\operatorname{lct}_{q}\left(S,\left.Z\right|_{S}\right) \geq \frac{2}{\sqrt{e_{q}\left(\left.Z\right|_{S}\right)}} \geq \frac{2}{r}>2 c
$$

by Proposition 2.13 (cf. Example 2.3). It follows that $\left(S,\left.2 c Z\right|_{S}\right)$ is $\log$ terminal near $q$. By inversion of adjunction, this implies that $\left(Y,\left.2 c Z\right|_{Y}\right)$ is $\log$ terminal near $q$. This proves that $\left(Y,\left.2 c Z\right|_{Y}\right)$ is $\log$ terminal away from a finite set $\Gamma$, as claimed.

Therefore the multiplier ideal $\mathcal{J}\left(Y,\left.2 c Z\right|_{Y}\right)$ defines a zero-dimensional subscheme $\Sigma \subset$ $Y$. Since $\left.Z\right|_{Y}$ is cut out by forms of degree $r$ and $2 c r<2$, we have $H^{1}\left(Y, \mathcal{O}_{Y}(2) \otimes\right.$ 
$\left.\mathcal{J}\left(Y,\left.2 c Z\right|_{Y}\right)\right)=0$ by Nadel's vanishing theorem (Theorem 2.14). This implies that there is a surjection $H^{0}\left(Y, \mathcal{O}_{Y}(2)\right) \rightarrow H^{0}\left(\Sigma, \mathcal{O}_{\Sigma}\right)$. Keeping in mind that $H^{0}\left(Y, \mathcal{O}_{Y}(2)\right) \cong$ $H^{0}\left(\mathbb{P}^{n}, \mathcal{O}_{\mathbb{P}^{n}}(2)\right)$, we obtain

$$
h^{0}\left(\Sigma, \mathcal{O}_{\Sigma}\right) \leq h^{0}\left(Y, \mathcal{O}_{Y}(2)\right)=\left(\begin{array}{c}
n+2 \\
2
\end{array}\right)
$$

On the other hand, Proposition 2.15 gives the lower-bound

$$
l\left(\mathcal{O}_{\Sigma}\right) \geq \frac{1}{2} 3^{n-1} .
$$

The two inequalities lead to a contradiction as soon as $n \geq 5$.

With the case $n=3$ already settled, this leaves open only the case $n=4$. We treat this case using generic projections, as in [dFEM03]. Note that the same argument can also be used to deal with the case $n=3$, instead of the argument based on Bezout's theorem we outlined earlier, hence one can think of the proof of Theorem 1.1 as splitting into two parts rather than three.

If $n=4$, then $Y$ is a threefold in $\mathbb{P}^{4}$ and $\left.Z\right|_{Y}$ is one dimensional. Let $f: Y \rightarrow \mathbb{P}^{2}$ be the map induced by a general linear projection $\mathbb{P}^{4} \rightarrow \mathbb{P}^{2}$. We may assume that the indeterminacies of $f$ are disjoint from the support of $\left.Z\right|_{Y}$, hence we can define the $\mathbb{Q}$-divisor

$$
\Delta=\frac{c^{2}}{4} \cdot f_{*}\left[\left.Z\right|_{Y}\right]
$$

Since the set $\left\{y \in Y \mid e_{y}\left(\left.Z\right|_{Y}\right)>r^{2}\right\}$ is zero dimensional and, for a general projection, $f$ restricts to a birational morphism on the support of $\left.Z\right|_{Y}$, it follows that the pair $\left(\mathbb{P}^{2}, \Delta\right)$ is log terminal in dimension one. By contrast, Theorem 2.19 implies that the pair is not $\log$ terminal at $f(p)$. Therefore, $\mathcal{J}\left(\mathbb{P}^{2}, \Delta\right)$ defines a zero dimensional scheme $W \subset \mathbb{P}^{2}$. Note that $\operatorname{deg}(\Delta)<2$. We have $H^{1}\left(\mathbb{P}^{2}, \mathcal{O}(-1) \otimes \mathcal{J}\left(\mathbb{P}^{2}, \Delta\right)\right)=0$ by Nadel's vanishing theorem, and this yields a surjection $H^{0}\left(\mathbb{P}^{2}, \mathcal{O}(-1)\right) \rightarrow H^{0}\left(\mathcal{O}_{W}\right)$, which is impossible since $H^{0}\left(\mathcal{O}_{W}\right) \neq\{0\}$.

Proof of Theorem 1.2. Let $X \subset \mathbb{P}^{n+1}$ be a hypersurface of degree $n+1$ with only isolated ordinary double points as singularities, and assume that $n \geq 5$. Note that $X$ is a Fano variety with terminal $\mathbb{Q}$-factorial singularities and $K_{X}$ generates the Picard group. It is easy to see that ordinary double points in dimension $\geq 3$ are terminal and locally factorial in the analytic topology, but $\mathbb{Q}$-factoriality is a local property in the Zariski topology, so it needs to be verified. This is done, for instance, in [dF17, Lemma 5.1].

The starting point of the proof of Theorem 1.2 is the same as in the smooth case just discussed. Assuming $X$ is not birationally superrigid, we construct a movable linear system $\mathcal{H} \subset\left|-r K_{X}\right|$ such that

$$
c:=\operatorname{ct}(X, \mathcal{H})<\frac{1}{r}
$$

Let $E$ be an exceptional divisor over $X$ such that $a_{E}(X, c \mathcal{H})=1$, and let $T \subset X$ be the center of $E$. We fix a general point $p \in T$. It follows by Proposition 2.8 and Proposition 2.13 (see also Remark 2.9) that $T$ is at most one-dimensional, and it is zero-dimensional if it is contained in the smooth locus of $X$. If $T \subset X_{\text {reg, }}$, then we can argue as in the proof of Theorem 1.1 to get a contradiction. We can therefore assume that $T \not \subset X_{\text {reg. }}$.

Let $Z=D \cap D^{\prime}$ be the intersection of two general members $D, D^{\prime} \in \mathcal{H}$ and $Y=V \cap V^{\prime} \subset$ $X$ the intersection of two general hyperplane sections $V, V^{\prime} \subset X$ through $p$. After cutting down with one hyperplane, we get a pair $\left(V,\left.c Z\right|_{V}\right)$ that is canonical in dimension 1. After cutting down with the second hyperplane, we get a pair $\left(Y,\left.c Z\right|_{Y}\right)$ that is not log canonical 
at $p$; here we apply inversion of adjunction. Furthermore, by Propositions 2.6 and 2.8 (see also Remark 2.9) we can ensure that the set $\left\{y \in Y \mid e_{y}\left(\left.Z\right|_{Y}\right)>r^{2}\right\}$ is zero-dimensional.

We split the proof in two cases, depending on the dimension of $X$.

We first treat the case where $n \geq 8$. By the same argument as in the proof of Theorem 1.1, we see that $\mathcal{J}\left(Y,\left.2 c Z\right|_{Y}\right)$ defines a zero dimensional scheme $\Sigma \subset Y$. Since $\left.Z\right|_{Y}$ is cut out on $Y$ by forms of degree $r$ and $2 c r<2$, we have $H^{1}\left(Y, \mathcal{O}_{Y}(3) \otimes \mathcal{J}\left(Y,\left.2 c Z\right|_{Y}\right)\right)=0$ by Nadel vanishing, hence

$$
h^{0}\left(\mathcal{O}_{\Sigma}\right) \leq h^{0}\left(Y, \mathcal{O}_{Y}(3)\right)=h^{0}\left(\mathbb{P}^{n-1}, \mathcal{O}(3)\right)=\left(\begin{array}{c}
n+2 \\
3
\end{array}\right) .
$$

By Lemma 2.16, we have $\operatorname{lct}(Y, \Sigma) \leq 1$. If $p$ is a smooth point, then we can apply Lemma 2.17 to get a lower bound. However, if $p$ is a singular point then we cannot apply the bound directly. Instead, we will take a suitable degeneration which will allow us to apply Lemma 2.17 in lower dimension.

Let us discuss here the case where $p$ is a singular point, the other case being easier and leading to a stronger bound. We restrict to affine chart $\mathbb{A}^{n-1}$ containing $p$, and fix coordinates $\left(x_{1}, \ldots, x_{n-1}\right)$ centered at $p$ such that the tangent cone of $Y$ at $p$ is defined by $\sum x_{i}^{2}=0$. For simplicity, we still denote by $Y$ its restriction to $\mathbb{A}^{n-1}$ and let $\Sigma$ denote now the connected component of the zero dimensional scheme defined by $\mathcal{J}\left(Y,\left.2 c Z\right|_{Y}\right)$ that is supported at $p$.

We take a flat degeneration of $Y \subset \mathbb{A}^{n-1}$ to the union $H_{1} \cup H_{2}$ of two hyperplanes through $p$, and let $\Sigma^{\prime} \subset \mathbb{A}^{n-1}$ be the zero dimensional scheme supported at $p$ obtained by flat degeneration from $\Sigma$. Note that $\Sigma^{\prime} \subset H_{1} \cup H_{2}$. Concretely, if $f\left(x_{1}, \ldots, x_{n-1}\right)=0$ is the equation of $Y$ in $\mathbb{A}^{n-1}$, then degeneration is constructed using the $\mathbb{G}_{m}$-action on $\mathbb{A}^{n-1}$ given by $x_{i} \mapsto \lambda x_{i}$ for $i=1,2$ and $x_{j} \mapsto \lambda^{2} x_{j}$ for $j>2$, and sending $\lambda \rightarrow 0$.

Recall that $\operatorname{mld}_{p}(Y, \Sigma) \leq 0$. By adjunction, this implies that

$$
\operatorname{mld}_{p}\left(\mathbb{A}^{n-1}, \Sigma+Y\right) \leq 0 .
$$

By semicontinuity of log canonical thresholds, we have

$$
\operatorname{mld}_{p}\left(\mathbb{A}^{n-1}, \Sigma^{\prime}+H_{1}+H_{2}\right) \leq 0 .
$$

By inversion of adjunction, this implies that

$$
\operatorname{mld}_{p}\left(H_{1}, \Sigma_{1}^{\prime}+H_{12}\right) \leq 0
$$

where $\Sigma_{1}^{\prime}=\Sigma^{\prime} \cap H_{1}$ and $H_{12}=H_{1} \cap H_{2}$. Applying inversion of adjunction again, we get

$$
\operatorname{mld}_{p}\left(H_{12}, \Sigma_{12}^{\prime}\right) \leq 0
$$

where $\Sigma_{12}^{\prime}=\Sigma_{1}^{\prime} \cap H_{12}=\Sigma^{\prime} \cap H_{12}$. Note that $H_{12}=\mathbb{A}^{n-3}$. Then Lemma 2.17 implies that

$$
l\left(\mathcal{O}_{\Sigma_{12}^{\prime}}\right) \geq \frac{1}{2} 3^{n-3} \text {. }
$$

Since $l\left(\mathcal{O}_{\Sigma}\right)=l\left(\mathcal{O}_{\Sigma^{\prime}}\right)$ by flatness and $l\left(\mathcal{O}_{\Sigma^{\prime}}\right) \geq l\left(\mathcal{O}_{\Sigma_{12}^{\prime}}\right)$ from the inclusion $\Sigma_{12}^{\prime} \subset \Sigma^{\prime}$, we conclude that

$$
l\left(\mathcal{O}_{\Sigma}\right) \geq \frac{1}{2} 3^{n-3} .
$$

Comparing the two inequalities (3.1) and (3.2), we get a contradiction as soon as $n \geq 8$. So the theorem is proved in this case.

We now address the remaining cases $5 \leq n \leq 7$. Suppose first that $\operatorname{dim} T=1$. Recall that we have fixed a general point $p \in T$ and let $Y:=V \cap V^{\prime} \subset X$ be the intersection of 
two general hyperplane sections through $p$. Let $f: Y \rightarrow \mathbb{P}^{n-3}$ be the map induced by a general linear projection $\mathbb{P}^{n-1} \rightarrow \mathbb{P}^{n-3}$, and let

$$
\Delta=\frac{c^{2}}{4} \cdot \pi_{*}\left[\left.Z\right|_{Y}\right]
$$

This is an effective $\mathbb{Q}$-divisor of degree less than $(n+1) / 4$. Since $\operatorname{deg}(\Delta)<2$, Nadel's vanishing theorem gives

$$
H^{1}\left(\mathbb{P}^{n-3}, \mathcal{O}(4-n) \otimes \mathcal{J}\left(\mathbb{P}^{n-3}, \Delta\right)\right)=0 .
$$

Theorem 2.19 implies that the pair $\left(\mathbb{P}^{n-3}, \Delta\right)$ is not log terminal at $f(p)$, and since the set $\left\{y \in Y \mid e_{y}\left(\left.Z\right|_{Y}\right)>r^{2}\right\}$ is zero dimensional, we can argue as in the proof of [dFEM03, Theorem 4.1] that if $n \in\{5,6\}$ then $\mathcal{J}\left(\mathbb{P}^{n-3}, \Delta\right)$ defines a zero dimensional scheme $W \subset$ $\mathbb{P}^{n-3}$, hence the surjection

$$
H^{0}\left(\mathbb{P}^{n-3}, \mathcal{O}(4-n)\right) \rightarrow H^{0}\left(\mathcal{O}_{W}\right)
$$

forces $n \leq 4$. If $n=7$, then one can only conclude that $\mathcal{J}\left(\mathbb{P}^{4}, \Delta\right)$ defines a scheme of dimension at most 1 , but after cutting down one more time and using the vanishing $H^{1}\left(\mathbb{P}^{3}, \mathcal{O}(-2) \otimes \mathcal{J}\left(\mathbb{P}^{3},\left.\Delta\right|_{\mathbb{P}^{3}}\right)\right)=0$ we get to a similar contradiction.

Therefore $T$ must be zero dimensional, i.e., $T=\{p\}$. With the case $p \in X_{\text {reg }}$ already been settled by the proof of Theorem 1.1, we assume that $p$ is a singular point. After cutting down with just one hyperplane section $V \subset X$ through $p$, we get $\operatorname{mld}_{p}\left(V,\left.c Z\right|_{V}\right) \leq 0$ by inversion of adjunction, hence we can find a divisor $E$ over $V$ with center $p$ such that $a_{E}\left(V,\left.c Z\right|_{V}\right) \leq 0$. Note that $\operatorname{Jac}_{V} \cdot \mathcal{O}_{V, p}=\mathfrak{m}_{V, p}$ since $p$ is an ordinary double point, hence

$$
\widehat{a}_{E}\left(V,\left.c Z\right|_{V}\right) \leq \operatorname{val}_{E}\left(\mathfrak{m}_{V, p}\right) .
$$

We take a general linear projection $g: V \rightarrow \mathbb{P}^{n-2}$ and let

$$
\Theta=\frac{c^{2}}{4} \cdot \pi_{*}\left[\left.Z\right|_{V}\right]
$$

By Theorem 2.19, there exists a divisor $G$ over $\mathbb{P}^{n-2}$ with center $q=g(p)$ such that,

$$
a_{G}\left(\mathbb{P}^{n-2}, \Theta\right) \leq \operatorname{val}_{G}\left(\mathfrak{m}_{q}\right)
$$

Restricting to a general hyperplane $\mathbb{P}^{n-3}$ through $q$ and letting $\Delta$ be the restriction of $\Theta$, we get a pair $\left(\mathbb{P}^{n-3}, \Delta\right)$ which satisfies the same properties as in the situation we discussed in the case $\operatorname{dim} T=1$. We can therefore repeat the same argument to get a contradiction.

This completes the proof of Theorem 1.2.

Remark 3.2. The first part of the proof of Theorem 1.2 works for more general quadratic singularities, with the same argument showing that, for $n \geq 8$, every hypersurface of degree $n+1$ in $\mathbb{P}^{n+1}$ with isolated quadratic singularities of rank $\geq 2$ is birationally superrigid. Note, however, that the part of the proof of Theorem 1.2 dealing with the case $5 \leq$ $n \leq 7$ does not extend automatically, as the Jacobian ideal at a quadratic singularity of submaximal rank is strictly contained in the maximal ideal and can have larger order along some valuations, invalidating the last step of the proof. 


\section{K-STABILITY}

K-stability is an algebro-geometric notion related to the existence of Kähler-Einstein metrics on Fano varieties. The original definition is given in terms of the positivity of the generalized Futaki invariant of test configurations. We will recall here an equivalent definition following the valuative approach of [Fuj19a,Li17]. According to the latter approach, an $n$-dimensional normal $\mathbb{Q}$-Gorenstein projective variety $X$ with ample anticanonical class is $K$-stable if for every divisor $E$ over $X$

$$
a_{E}(X)>\frac{1}{\left(-K_{X}\right)^{n}} \int_{0}^{\infty} \operatorname{vol}\left(f^{*}\left(-K_{X}\right)-t E\right) d t
$$

where $f: Y \rightarrow X$ is a resolution on which $E$ appears as a divisor. This definition better fits the theme of this paper. The equivalence between this definition and the one via the generalized Futaki invariant is proved in [BX19]. We refer to [Xu20] for a general introduction to the subject.

We use the following result, due to [SZ19]. A stronger result along the same lines is proved in [Zhu20], but we will not need it.

Theorem 4.1. Let $X$ be a Fano variety with $\mathbb{Q}$-factorial terminal singularities and Picard number 1. Assume that $X$ is birationally superrigid and $\operatorname{lct}(X, D)>\frac{1}{2}$ for every effective $\mathbb{Q}$-divisor $D \sim_{\mathbb{Q}}-K_{X}$. Then $X$ is K-stable.

As it is already observed in [SZ19], in view of Theorem 1.1 and well-established bounds on the alpha invariant of smooth Fano hypersurfaces, this result implies immediately that smooth hypersurfaces of degree $n+1$ in $\mathbb{P}^{n+1}$ are $K$-stable hence admit a Kähler-Einstein metric. In order to apply the result to hypersurfaces with isolated ordinary double points, we need the following property.

Proposition 4.2. For any $n \geq 4$, if $X \subset \mathbb{P}^{n+1}$ is a hypersurface of degree $n+1$ with only isolated ordinary double points as singularities, then $\operatorname{lct}(X, D)>\frac{1}{2}$ for every effective $\mathbb{Q}$-divisor $D \sim \mathbb{Q}-K_{X}$.

Proof. Suppose by contradiction that there exists an effective $\mathbb{Q}$-divisor $D \sim_{\mathbb{Q}}-K_{X}$ such that $\operatorname{lct}(X, D) \leq \frac{1}{2}$. Let $E$ be a divisor over $X$ such that $a_{E}\left(X, \frac{1}{2} D\right) \leq 0$, and let $T \subset X$ be the center of $E$ in $X$. Propositions 2.8 and 2.13 imply that $T$ is at most one-dimensional, and that it is zero-dimensional if it is contained in the smooth locus of $X$. Let $p \in T$ be a general point.

Let $f: X \rightarrow \mathbb{P}^{n}$ be induced by a general linear projection, let $q=f(p)$, and let

$$
\Delta=\frac{1}{2} \cdot f_{*} D
$$

We fix a general hyperplane $\mathbb{P}^{n-1} \subset \mathbb{P}^{n}$ through $q$.

We claim that

$$
\operatorname{mld}_{q}\left(\mathbb{P}^{n-1},\left.\Delta\right|_{\mathbb{P}^{n-1}}\right) \leq 0 .
$$

If $\operatorname{dim} T=1$ and $\xi$ is the generic point of $f(T)$, then we have $\operatorname{mld}_{\xi}\left(\mathbb{P}^{n}, \Delta\right) \leq 0$ by Theorem 2.19, and since $q$ is a general point of $f(T),(4.1)$ follows by Bertini's theorem applied on a $\log$ resolution. If $T=\{p\}$ is zero-dimensional, then we have $\widehat{a}_{E}\left(X, \frac{1}{2} D\right) \leq \operatorname{val}_{E}\left(\mathfrak{m}_{p}\right)$. This is clear if $p \in X_{\text {reg }}$, and holds if $p$ is a singular point since in this case we have $\operatorname{Jac}_{X} \cdot \mathcal{O}_{X, p}=\mathfrak{m}_{p}$. Then Theorem 2.19 implies that there is a divisor $F$ over $\mathbb{P}^{n}$ with center $q$ such that $a_{F}\left(\mathbb{P}^{n}, \Delta\right) \leq \operatorname{val}_{F}\left(\mathfrak{m}_{q}\right)$. This implies that $\operatorname{mld}_{q}\left(\mathbb{P}^{n}, \Delta+\mathbb{P}^{n-1}\right) \leq 0$, hence (4.1) follows by inversion of adjunction. 
Our next claim is that the pair $\left(\mathbb{P}^{n-1},\left.\Delta\right|_{\mathbb{P}^{n-1}}\right)$ is $\log$ terminal in dimension 1 near $q$. We will prove this by showing that the pair $\left(\mathbb{P}^{n}, \Delta\right)$ is log terminal in dimension 2 near $q$. To see this, we restrict to formal neighborhoods. Let $U=\operatorname{Spec}\left(\widehat{O_{X, p}}\right)$ and $V=\operatorname{Spec}\left(\widehat{\mathcal{O}_{\mathbb{P}^{n}, q}}\right)$, and denote by $g=\left.f\right|_{U}: U \rightarrow V$ the induced map. Resolution of singularities holds in this setting (and in fact for the purpose of this proof it suffice to rely solely on resolutions obtained by base-change from $X$ and $\mathbb{P}^{n}$, respectively), so we can extend the definition of singularities to this setting. We are going to show that $\left(V,\left.\Delta\right|_{V}\right)$ is log terminal in dimension 2. By taking a general projection we can ensure that $p$ is the only point in the support of $D$ mapping to $q$, hence $\left.\Delta\right|_{V}=\frac{1}{2} \cdot g_{*}\left(\left.D\right|_{U}\right)$. If $p$ is a smooth point of $X$, then $g$ is an isomorphism and the claim is clear. If $p$ is a singular point, then $g$ is a generically two-to-one cover. In this case, if $G$ is any divisor over $V$ with positive dimensional center $W \subset V$, and $G^{\prime}$ is any divisor over $U$ such that $\operatorname{val}_{G^{\prime}}$ restricts to $b \operatorname{val}_{G}$ on $V$ for some $b \in \mathbb{N}$ (such $G^{\prime}$ always exists), then a standard computation gives

$$
b a_{G}\left(V,\left.\Delta\right|_{V}\right)=a_{G^{\prime}}\left(U, g^{*}\left(\left.\Delta\right|_{V}\right)-K_{U / V}\right) \geq a_{G^{\prime}}\left(U, g^{*}\left(\left.\Delta\right|_{V}\right)\right) .
$$

Here we are using the fact that $W$ is positive dimensional in order to reduce to a computation on smooth varieties (i.e., away from $p$ and $q$ ), and use that $K_{U / V} \geq 0$ in the last inequality. If $\tau: U \rightarrow U$ is the involution defined by the cover $U \rightarrow V$, then $g^{*}\left(\left.\Delta\right|_{V}\right)=\frac{1}{2}\left(\left.D\right|_{U}+\tau\left(\left.D\right|_{U}\right)\right)$, hence

$$
\operatorname{val}_{G^{\prime}}\left(g^{*}\left(\left.\Delta\right|_{V}\right)\right) \leq \max \left\{\operatorname{val}_{G^{\prime}}\left(\left.D\right|_{U}\right), \operatorname{val}_{G^{\prime}}\left(\tau\left(\left.D\right|_{U}\right)\right)\right\} .
$$

Since both $\left(U,\left.D\right|_{U}\right)$ and $\left(U, \tau\left(\left.D\right|_{U}\right)\right)$ are log terminal in dimension 2, it follows that $a_{G^{\prime}}\left(U, g^{*}\left(\left.\Delta\right|_{V}\right)\right)>0$, and hence $a_{G}\left(V,\left.\Delta\right|_{V}\right)>0$ by $(4.2)$, whenever $\operatorname{dim}(W) \geq 2$. This proves that $\left(V,\left.\Delta\right|_{V}\right)$ is $\log$ terminal in dimension 2 , which in turns implies that $\left(\mathbb{P}^{n}, \Delta\right)$ is $\log$ terminal in dimension 2 near $q$. Since $\mathbb{P}^{n-1}$ is a general hyperplane through $q$, we conclude that $\left(\mathbb{P}^{n-1},\left.\Delta\right|_{\mathbb{P}^{n-1}}\right)$ is log terminal in dimension 1 near $q$.

We are now ready to finish the proof. Since $\operatorname{deg}(\Delta)<\left\lceil\frac{n}{2}\right\rceil+1$, Nadel's vanishing gives

$$
H^{1}\left(\mathbb{P}^{n-1}, \mathcal{O}\left(-n+\left\lceil\frac{n}{2}\right\rceil+1\right) \otimes \mathcal{J}\left(\mathbb{P}^{n-1},\left.\Delta\right|_{\mathbb{P}^{n-1}}\right)\right)=0 .
$$

Note that $-n+\left\lceil\frac{n}{2}\right\rceil+1 \leq-1$ for $n \geq 4$. As the multiplier ideal $\mathcal{J}\left(\mathbb{P}^{n-1},\left.\Delta\right|_{\mathbb{P} n-1}\right)$ defines a scheme with a zero-dimensional component supported at $q$, this gives a contradiction.

Proof of Theorem 1.3. It follows by Proposition 4.2 and Theorem 4.1 that $X$ is K-stable, and [LXZ21, Theorem 1.5] implies that $X$ admits a weak Kähler-Einstein metric.

Remark 4.3. Proposition 4.2 also holds for $n=4$, a case which is not covered by Theorem 1.2. If one can extend Theorem 1.2 to include the four-dimensional case, then Kstability would follow as well.

\section{REFERENCES}

[Ben70] B. M. Bennett, On the characteristic functions of a local ring, Ann. of Math. 91 (1970), 25-87. $\uparrow 3$

[BX19] Harold Blum and Chenyang Xu, Uniqueness of K-polystable degenerations of Fano varieties, Ann. of Math. (2) 190 (2019), no. 2, 609-656. $\uparrow 12$

[Che01] Ivan Cheltsov, Log canonical thresholds on hypersurfaces, Mat. Sb. 192 (2001), no. 8, 155-172 (Russian, with Russian summary); English transl., Sb. Math. 192 (2001), no. 7-8, 1241-1257. $\uparrow 2$

[Che07] _ On nodal sextic fivefold, Math. Nachr. 280 (2007), no. 12, 1344-1353. $\uparrow 1$ 
[Cor00] Alessio Corti, Singularities of linear systems and 3-fold birational geometry, Explicit birational geometry of 3-folds, London Math. Soc. Lecture Note Ser., vol. 281, Cambridge Univ. Press, Cambridge, 2000, pp. 259-312. $\uparrow 1,7$

[CM04] Alessio Corti and Massimiliano Mella, Birational geometry of terminal quartic 3-folds. I, Amer. J. Math. 126 (2004), no. 4, 739-761. $\uparrow 1$

[dF13] Tommaso de Fernex, Birationally rigid hypersurfaces, Invent. Math. 192 (2013), no. 3, 533-566. $\uparrow 3,5$

[dF16] Erratum to: Birationally rigid hypersurfaces, Invent. Math. 203 (2016), no. 2, 675-680. $\uparrow 1$

[dF17] , Birational rigidity of singular Fano hypersurfaces, Ann. Sc. Norm. Super. Pisa Cl. Sci. (5) 17 (2017), no. 3, 911-929. $\uparrow 2,9$

[dFD14] Tommaso de Fernex and Roi Docampo, Jacobian discrepancies and rational singularities, J. Eur. Math. Soc. (JEMS) 16 (2014), no. 1, 165-199. $\uparrow 6$

[dFEM03] Tommaso de Fernex, Lawrence Ein, and Mircea Mustaţă, Bounds for log canonical thresholds with applications to birational rigidity, Math. Res. Lett. 10 (2003), no. 2-3, 219-236. 1 , 2, 3, 4, $7,9,11$

[dFEM04] $\underset{\uparrow 1,5,6}{\longrightarrow}$, Multiplicities and log canonical threshold, J. Algebraic Geom. 13 (2004), no. 3, 603-615.

[dFM15] Tommaso de Fernex and Mircea Mustaţă, The volume of a set of arcs on a variety, Rev. Roumaine Math. Pures Appl. 60 (2015), 375-401. Special issue in honor of Lucian Badescu's 70th birthday. $\uparrow 2,7$

[EP14] Thomas Eckl and Aleksandr Pukhlikov, On the locus of nonrigid hypersurfaces, Automorphisms in birational and affine geometry, Springer Proc. Math. Stat., vol. 79, Springer, Cham, 2014. $\uparrow 1$

[Fan07] Gino Fano, Sopra alcune varietà algebriche a tre dimensioni aventi tutti $i$ generi nulli, Atti Accad. Torino 43 (1907/08), 973-377. $\uparrow 1$

[Fan15] — Osservazioni sopra alcune varietà non razionali aventi tutti i generi nulli, Atti Accad. Torino 47 (1915), 1067-1071. $\uparrow 1$

[Fuj19a] Kento Fujita, A valuative criterion for uniform $K$-stability of $\mathbb{Q}$-Fano varieties, J. Reine Angew. Math. 751 (2019), 309-338. $\uparrow 12$

[Fuj19b] , K-stability of Fano manifolds with not small alpha invariants, J. Inst. Math. Jussieu 18 (2019), no. 3, 519-530. $\uparrow 2$

[Ful98] William Fulton, Intersection theory, 2nd ed., Ergebnisse der Mathematik und ihrer Grenzgebiete. 3. Folge. A Series of Modern Surveys in Mathematics [Results in Mathematics and Related Areas. 3rd Series. A Series of Modern Surveys in Mathematics], vol. 2, Springer-Verlag, Berlin, 1998. $\uparrow 3,4$

[IM71] V. A. Iskovskih and Ju. I. Manin, Three-dimensional quartics and counterexamples to the Lüroth problem, Mat. Sb. (N.S.) 86(128) (1971), 140-166 (Russian). $\uparrow 1,7$

[Kol92] János Kollár, Adjunction and discrepancies, Flips and abundance for algebraic threefolds, 1992, pp. 1-258. Papers from the Second Summer Seminar on Algebraic Geometry held at the University of Utah, Salt Lake City, Utah, August 1991; Astérisque No. 211 (1992) (1992). $\uparrow 5$

[Kol19] _ The rigidity theorem of Fano-Segre-Iskovskikh-Manin-Pukhlikov-Corti-Cheltsov-de Fernex-Ein-Mustaţă-Zhuang, Birational Geometry of Hypersurfaces, Gargnano del Garda, Italy, 2018, Lecture Notes of the Unione Matematica Italiana, Springer International Publishing, 2019. $\uparrow 1,5,8$

[Laz04] Robert Lazarsfeld, Positivity in algebraic geometry. II, Ergebnisse der Mathematik und ihrer Grenzgebiete. 3. Folge. A Series of Modern Surveys in Mathematics [Results in Mathematics and Related Areas. 3rd Series. A Series of Modern Surveys in Mathematics], vol. 49, SpringerVerlag, Berlin, 2004. Positivity for vector bundles, and multiplier ideals. $\uparrow 5$

[Li17] Chi Li, K-semistability is equivariant volume minimization, Duke Math. J. 166 (2017), no. 16, 3147-3218. $\uparrow 12$

[LZ19] Yuchen Liu and Ziquan Zhuang, On the sharpness of Tian's criterion for K-stability (2019). Preprint, arXiv: 1903.04719. $\uparrow 2$

[LZ21] - Birational superrigidity and K-stability of singular Fano complete intersections, Int. Math. Res. Not. IMRN 1 (2021), 384-403. $\uparrow 2$

[LXZ21] Yuchen Liu, Chenyang Xu, and Ziquan Zhuang, Finite generation for valuations computing stability thresholds and applications to K-stability (2021). Preprint, arXiv:2102.09405. $\uparrow 2,13$ 
[Man66] Ju. I. Manin, Rational surfaces over perfect fields, Inst. Hautes Études Sci. Publ. Math. 30 (1966), 55-113 (Russian, with English summary). $\uparrow 1$

[Mel04] Massimiliano Mella, Birational geometry of quartic 3-folds. II. The importance of being $\mathbb{Q}-$ factorial, Math. Ann. 330 (2004), no. 1, 107-126. $\uparrow 1$

[Nob75] A. Nobile, Some properties of the Nash blowing-up, Pacific J. Math. 60 (1975), no. 1, $297-305$. $\uparrow 6$

[OO13] Yuji Odaka and Takuzo Okada, Birational superrigidity and slope stability of Fano manifolds, Math. Z. 275 (2013), no. 3-4, 1109-1119. $\uparrow 2$

[OS12] Yuji Odaka and Yuji Sano, Alpha invariant and K-stability of $\mathbb{Q}$-Fano varieties, Adv. Math. 229 (2012), no. 5, 2818-2834. $\uparrow 2$

[Pie79] Ragni Piene, Ideals associated to a desingularization, Algebraic geometry (Proc. Summer Meeting, Univ. Copenhagen, Copenhagen, 1978), Lecture Notes in Math., vol. 732, Springer, Berlin, 1979, pp. 503-517. $\uparrow 7$

[Puk87] A. V. Pukhlikov, Birational isomorphisms of four-dimensional quintics, Invent. Math. 87 (1987), no. $2,303-329 . \uparrow 1$

[Puk88] - Birational automorphisms of a three-dimensional quartic with a simple singularity, Mat. Sb. (N.S.) 135(177) (1988), no. 4, 472-496, 559 (Russian); English transl., Math. USSR-Sb. 63 (1989), no. $2,457-482 . \uparrow 1$

[Puk98] _ Birational automorphisms of Fano hypersurfaces, Invent. Math. 134 (1998), no. 2, 401426. $\uparrow 1$

[Puk02a] _ Birationally rigid Fano hypersurfaces, Izv. Ross. Akad. Nauk Ser. Mat. 66 (2002), no. 6, 159-186 (Russian, with Russian summary); English transl., Izv. Math. 66 (2002), no. 6, $1243-$ 1269. $\uparrow 4$

[Puk02b] _ Birationally rigid Fano hypersurfaces with isolated singularities, Mat. Sb. 193 (2002), no. 3, 135-160 (Russian, with Russian summary); English transl., Sb. Math. 193 (2002), no. 3-4, 445-471. $\uparrow 1$

[Puk05] _ Birational geometry of Fano direct products, Izv. Ross. Akad. Nauk Ser. Mat. 69 (2005), no. 6, 153-186 (Russian, with Russian summary); English transl., Izv. Math. 69 (2005), no. 6, 1225-1255. $\uparrow 2$

[Puk21] _ Birational geometry of varieties, fibred into complete intersections of codimension two (2021). Preprint, arXiv:2101.10830. $\uparrow 2$

[Puk15] _ Birationally rigid Fano fibrations. II, Izv. Ross. Akad. Nauk Ser. Mat. 79 (2015), no. 4, 175-204 (Russian, with Russian summary); English transl., Izv. Math. 79 (2015), no. 4, 809-837. $\uparrow 2$

[Seg51] Beniamino Segre, On the rational solutions of homogeneous cubic equations in four variables, Math. Notae 11 (1951), 1-68. $\uparrow 1$

[Sho92] V. V. Shokurov, Three-dimensional log perestroikas, Izv. Ross. Akad. Nauk Ser. Mat. 56 (1992), no. 1, 105-203 (Russian); English transl., Russian Acad. Sci. Izv. Math. 40 (1993), no. 1, 95-202. $\uparrow 5$

[SZ19] Charlie Stibitz and Ziquan Zhuang, K-stability of birationally superrigid Fano varieties, Compos. Math. 155 (2019), no. 9, 1845-1852. ^2, 12

[Tia87] Gang Tian, On Kähler-Einstein metrics on certain Kähler manifolds with $C_{1}(M)>0$, Invent. Math. 89 (1987), no. 2, 225-246. $\uparrow 2$

[Xu20] Chenyang Xu, K-stability of Fano varieties: an algebro-geometric approach (2020). Preprint, arXiv:2011.10477. $\uparrow 12$

[Zhu20] Ziquan Zhuang, Birational superrigidity and K-stability of Fano complete intersections of index 1, Duke Math. J. 169 (2020), no. 12, 2205-2229. With an appendix by Zhuang and Charlie Stibitz. $\uparrow 1,2,5,8,12$

Department of Mathematics, University of Utah, Salt Lake City, UT 48112, USA

Email address: defernex@math.utah.edu 